\title{
Asteroid (16) Psyche’s primordial shape: A possible Jacobi ellipsoid ${ }^{\star, \star \star}$
}

\author{
M. Ferrais ${ }^{1}$, P. Vernazza ${ }^{1}$, L. Jorda ${ }^{1}$, N. Rambaux ${ }^{2}$, J. Hanuš ${ }^{3}$, B. Carry ${ }^{4}$, F. Marchis ${ }^{1,5}$, M. Marsset ${ }^{6}$,
} M. Viikinkoski ${ }^{7}$, M. Brož ${ }^{3}$, R. Fetick ${ }^{1}$, A. Drouard ${ }^{1}$, T. Fusco ${ }^{1}$, M. Birlan ${ }^{2,8}$, E. Podlewska-Gaca ${ }^{9}, 10$, E. Jehin ${ }^{11}$, P. Bartczak ${ }^{9}$, J. Berthier ${ }^{2}$, J. Castillo-Rogez ${ }^{12}$, F. Cipriani ${ }^{13}$, F. Colas ${ }^{2}$, G. Dudziński ${ }^{9}$, C. Dumas ${ }^{14}$, J. Durech ${ }^{3}$, M. Kaasalainen ${ }^{7}$, A. Kryszczynska ${ }^{9}$, P. Lamy ${ }^{1}$, H. Le Coroller ${ }^{1}$, A. Marciniak ${ }^{9}$, T. Michalowski ${ }^{9}$, P. Michel ${ }^{4}$, T. Santana-Ros ${ }^{15,16}$, P. Tanga ${ }^{4}$, F. Vachier ${ }^{2}$, A. Vigan ${ }^{1}$, O. Witasse ${ }^{14}$, and B. Yang ${ }^{17}$

1 Aix Marseille Université, CNRS, CNES, Laboratoire d'Astrophysique de Marseille, Marseille, France e-mail: marin.ferrais@lam.fr

2 IMCCE, CNRS, Observatoire de Paris, PSL Université, Sorbonne Université, Paris, France

3 Institute of Astronomy, Charles University, Prague, V Holešovičkách 2, 18000 Prague 8, Czech Republic

${ }^{4}$ Université Côte d'Azur, Observatoire de la Côte d'Azur, CNRS, Laboratoire Lagrange, France

5 SETI Institute, Carl Sagan Center, 189 Bernado Avenue, Mountain View, CA 94043, USA

6 Department of Earth, Atmospheric and Planetary Sciences, MIT, 77 Massachusetts Avenue, Cambridge, MA 02139, USA

7 Mathematics and Statistics, Tampere University, 33720 Tampere, Finland

Astronomical Institute of the Romanian Academy, 5-Cuţitul de Argint, 040557 Bucharest, Romania

Astronomical Observatory Institute, Faculty of Physics, Adam Mickiewicz University, Słoneczna 36, 60-286 Poznań, Poland

${ }^{10}$ Institute of Physics, University of Szczecin, Wielkopolska 15, 70-453 Szczecin, Poland

1 Space sciences, Technologies and Astrophysics Research Institute, Université de Liège, Allée du 6 Août 17, 4000 Liège, Belgium

12 Jet Propulsion Laboratory, California Institute of Technology, 4800 Oak Grove Drive, Pasadena, CA 91109, USA

13 European Space Agency, ESTEC - Scientific Support Office, Keplerlaan 1, Noordwijk 2200 AG, The Netherlands

14 TMT Observatory, 100 W. Walnut Street, Suite 300, Pasadena, CA 91124, USA

15 Departamento de Fisica, Ingeniería de Sistemas y Teoría de la Señal, Universidad de Alicante, Alicante, Spain

${ }^{16}$ Institut de Ciències del Cosmos (ICCUB), Universitat de Barcelona (IEEC-UB), Martí Franqués 1, E08028 Barcelona, Spain

17 European Southern Observatory (ESO), Alonso de Cordova 3107, 1900 Casilla Vitacura, Santiago, Chile

Received 6 April 2020 / Accepted 2 June 2020

\section{ABSTRACT}

Context. Asteroid (16) Psyche is the largest M-type asteroid in the main belt and the target of the NASA Psyche mission. It is also the only asteroid of this size $(D>200 \mathrm{~km})$ known to be metal rich. Although various hypotheses have been proposed to explain the rather unique physical properties of this asteroid, a perfect understanding of its formation and bulk composition is still missing.

Aims. We aim to refine the shape and bulk density of (16) Psyche and to perform a thorough analysis of its shape to better constrain possible formation scenarios and the structure of its interior.

Methods. We obtained disk-resolved VLT/SPHERE/ZIMPOL images acquired within our ESO large program (ID 199.C-0074), which complement similar data obtained in 2018. Both data sets offer a complete coverage of Psyche's surface. These images were used to reconstruct the three-dimensional (3D) shape of Psyche with two independent shape modeling algorithms (MPCD and ADAM). A shape analysis was subsequently performed, including a comparison with equilibrium figures and the identification of mass deficit regions.

Results. Our 3D shape along with existing mass estimates imply a density of $4.20 \pm 0.60 \mathrm{~g} \mathrm{~cm}^{-3}$, which is so far the highest for a solar system object following the four telluric planets. Furthermore, the shape of Psyche presents small deviations from an ellipsoid, that is, prominently three large depressions along its equator. The flatness and density of Psyche are compatible with a formation at hydrostatic equilibrium as a Jacobi ellipsoid with a shorter rotation period of $\sim 3 \mathrm{~h}$. Later impacts may have slowed down Psyche's rotation, which is currently $\sim 4.2 \mathrm{~h}$, while also creating the imaged depressions.

Conclusions. Our results open the possibility that Psyche acquired its primordial shape either after a giant impact while its interior was already frozen or while its interior was still molten owing to the decay of the short-lived radionuclide ${ }^{26} \mathrm{Al}$.

Key words. minor planets, asteroids: general - minor planets, asteroids: individual: (16) Psyche - methods: observational techniques: high angular resolution

\footnotetext{
$\star$ The reduced images are only available at the CDS via anonymous ftp to cdsarc.u-strasbg.fr (130.79.128.5) or via http://cdsarc.u-strasbg.fr/viz-bin/cat/J/A+A/638/L15

$\star \star$ Based on observations made with ESO Telescopes at the Paranal Observatory under programme ID 199.C-0074 (PI: P. Vernazza).
} 


\section{Introduction}

Asteroid (16) Psyche is the largest M-type asteroid in the main belt. This asteroid was defined as a metallic world (ElkinsTanton et al. 2017) resulting from compelling evidences of a metal-rich surface, including a high mean radar albedo $(0.37$; Shepard et al. 2017) and a thermal inertia among the highest for an asteroid of this size (Matter et al. 2013). The uniqueness of Psyche's characteristics and the hypothesis that it could be an exposed planetary core made it a prime target for spacecraft exploration. This was concretized by its selection as the rendezvous target of a NASA Discovery mission that is scheduled to arrive at Psyche in early 2026 (Elkins-Tanton et al. 2017).

To this day, the true nature of Psyche, the only metalrich asteroid with a diameter above $200 \mathrm{~km}$, remains a mystery that leads to the formulation of several distinct formation scenarios. One of these scenarios involves hit-and-run collisions exposing the core of a differentiated planetesimal, such as Vesta, in the early history of the solar system (Asphaug et al. 2006; Asphaug \& Reufer 2014). However, recent estimates of Psyche's density all agree on a value close to $\sim 4 \mathrm{~g} \mathrm{~cm}^{-3}$ (Shepard et al. 2017; Hanuš et al. 2017; Drummond et al. 2018; Viikinkoski et al. 2018), much lower than the density of iron meteorites $\left(\sim 7.5 \mathrm{~g} \mathrm{~cm}^{-3}\right)$. A high macroporosity of $\sim 50 \%$, if Psyche is entirely composed of metal, would solve this discrepancy, but appears unlikely considering the low macroporosity (typically less than 20\%) typical of asteroids with masses comparable or greater than Psyche's $\left(m>10^{19} \mathrm{~kg}\right.$; Carry 2012; Scheeres et al. 2015; Carry et al. 2019). Alternatively, Psyche's density appears compatible with that of stony-iron meteorites such as mesosiderites (Viikinkoski et al. 2018) and pallasites (Elkins-Tanton et al. 2020) as well as that of CB chondrites (Elkins-Tanton et al. 2020). Recently, Johnson et al. (2020) proposed that Psyche formed as a differentiated body and that ferrovolcanism could be at the origin of the high metal content of its surface (Shepard et al. 2017). As of today, it is not understood whether Psyche formed as a differentiated or undifferentiated body.

With the renew of interest in Psyche since its selection as the target of a NASA mission, recent observations suggest that the nature of Psyche might not be a metallic world as initially defined but rather a mix of metal and silicates. A review of the possible compositions, meteorite analogs, and formation and evolution scenarios of Psyche can be found in Elkins-Tanton et al. (2020).

In this paper, we present new high angular resolution imaging observations of Psyche acquired in the framework of our ESO large program (PI: P. Vernazza; ID: 199.C-0074) with the extreme adaptive optics instrument VLT/SPHERE. These new images were used along with those obtained in 2018 (Viikinkoski et al. 2018) to (i) reconstruct Psyche's threedimensional (3D) shape using two different modeling techniques and thus to refine its density and spin axis orientation; (ii) perform a detailed analysis of its shape, including a comparison with that of other large asteroids; and (iii) more generally make progress in our understanding of its origin and formation.

\section{Observations}

We obtained 35 images of Psyche at 7 epochs in July and August 2019 using VLT/SPHERE/ZIMPOL (Beuzit et al. 2008; Thalmann et al. 2008). This data set complements the first 25 images taken within our ESO large program that was already presented in Viikinkoski et al. (2018), for a total of 60 images taken at 12 epochs. All images were reduced following the procedure described in Vernazza et al. (2018) and were subsequently deconvolved with the Mistral algorithm (Fusco et al. 2003; Mugnier et al. 2004). Details about the deconvolution procedure can be found in Fétick et al. (2019).

We selected two to three images per epoch, maximizing the time gap between the images, instead of using all of the images which would not have added more information; the criterion is a difference of at least one pixel between two images owing to the asteroid rotation. The observational circumstances of the images acquired in 2019 are presented in Table A.1 and the deconvolved images are shown in Fig. A.1.

Whereas the images acquired during the first observation campaign (Viikinkoski et al. 2018) were limited to the northern hemisphere of Psyche (sub-observer latitude of $\sim 75^{\circ}$ ), those acquired during the second apparition covered well the equatorial region ("edge-on") with a sub-observer latitude of $\sim-10^{\circ}$. This allowed us to obtain a complete coverage of the surface of Psyche, the southern hemisphere being observed everywhere by a minimum of six images (see the coverage map, Fig. A.2). Moreover, whereas the "pole-on" apparition allowed us to obtain robust constraints on the dimensions along the $x$ and $y$ axes, the new "equator-on" apparition was crucial to constrain the $z$-axis dimension and thus to refine Psyche's volume.

\section{Methods: Three-dimensional shape reconstruction}

First, an updated shape model of Psyche was generated with the All-Data Asteroid Modeling (ADAM) algorithm (Viikinkoski et al. 2015) using the same procedure as in Viikinkoski et al. (2018). In addition to the new VLT/SPHERE images, a new stellar occultation by Psyche recorded in October 2019 was included in the modeling (Fig. A.3), whereas two previous occultations (Dunham et al. 2017) were only used for shape validation. The shape was again optimized simultaneously with the albedo variegation apparent both in the light curves and the images. The derived relative albedo distribution is shown in Fig. A.4.

We then applied the so-called Multi-resolution PhotoClinometry by Deformation (MPCD) method (Capanna et al. 2013; Jorda et al. 2016) on our sample of 35 VLT/SPHERE/ZIMPOL images from 12 different epochs to reconstruct the 3D shape of Psyche. This method gradually deforms the vertices of an initial mesh to minimize the difference between the observed images and synthetic images of the deformed model created with the OASIS software (Optimized Astrophysical Simulator for Imaging Systems; Jorda et al. 2010). The OASIS software uses a shape model described by a triangular mesh along with geometric and radiometric parameters to generate realistic images of the surface. As inputs, this software takes the position and orientation of the target in the camera frame, the radiometric properties of the camera, the rotational parameter of the object, and the reflectance properties of its surface. The MPCD method has already been successfully applied to images of small bodies observed by the Rosetta mission, notably those of comet $67 \mathrm{P}$ (Jorda et al. 2016).

For the rotational parameters of our model, we first used the pole solution of ADAM and its sidereal rotation period of 4.195948 h. We also adopted the ADAM shape model as the initial model for our reconstruction. For the reflectance properties of the surface, we used the five-parameter Hapke law (Hapke 1981) derived for another M-type asteroid, (216) Kleopatra (Descamps et al. 2011). This choice had little influence on the shape reconstruction because of the low phase angle of our observations 


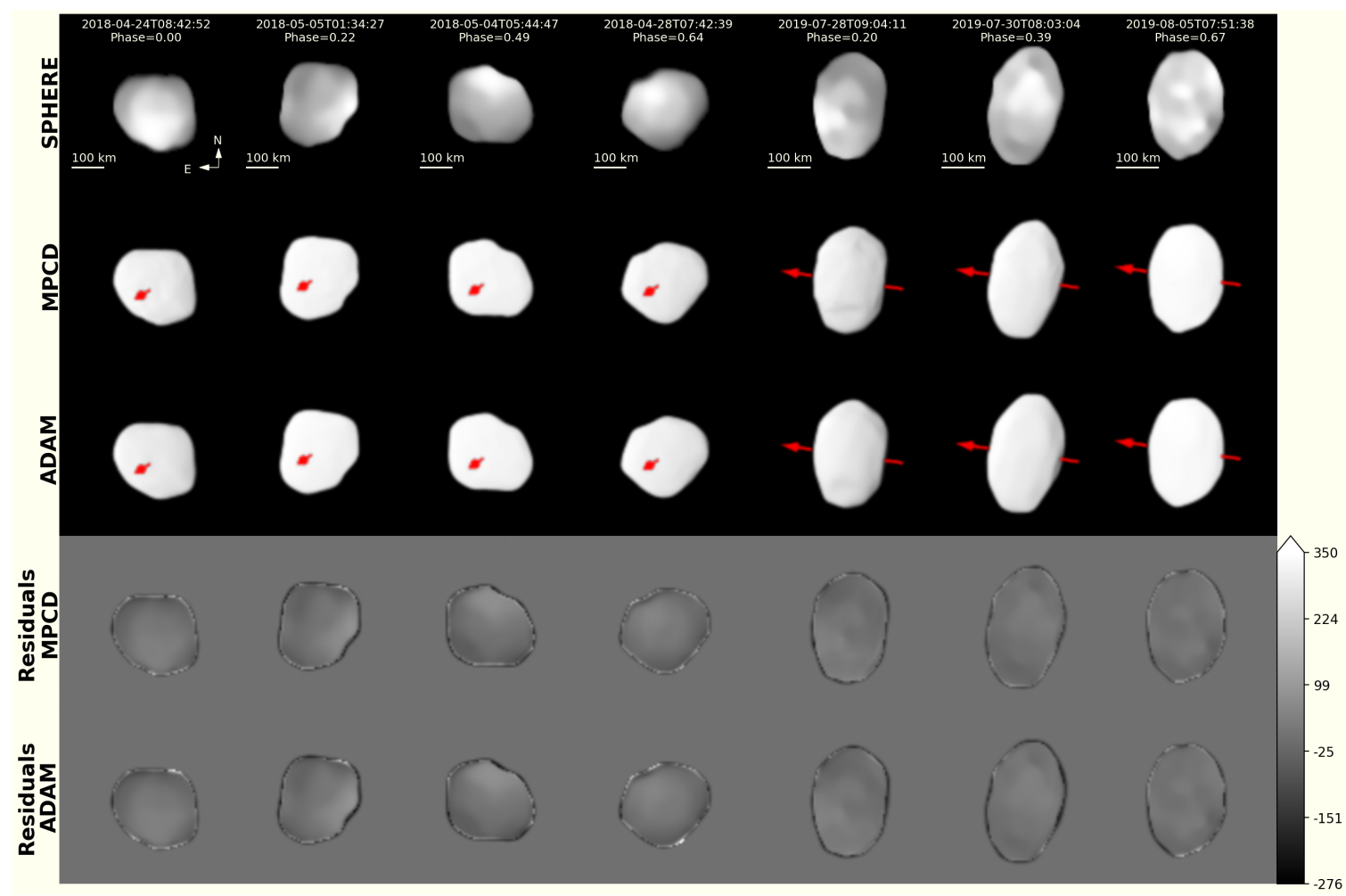

Fig. 1. Comparison between VLT/SPHERE/ZIMPOL deconvolved images of Psyche (top row) and the corresponding synthetic images generated by OASIS of our MPCD (second row) and ADAM (third row) shape models. The red arrows indicate the direction of the spin axis. Fourth and fifth rows: residuals in units of instrumental noise between the observed images and the synthetic images of the MPCD and ADAM shape models, respectively. Given that all images were taken close to opposition with a phase angle less than $9^{\circ}$, the color variation across the disk reflects a variation of albedo rather than a variation in topography. A map of the albedo variegation is shown in Fig. A.4.

$\left(\alpha_{\max }=8.7^{\circ}\right)$. This was verified by testing the Hapke parameters of other asteroid types, resulting in similar shape models. The best point-spread function (PSF) to use for the convolution of the synthetic images was determined by testing a range of Gaussian PSF on the ADAM model. The PSF resulting in the lowest $\chi^{2}$ had a full width at half maximum (FWHM) of 2.1 pixels (7.6" $\left.{ }^{\prime \prime}\right)$.

The first run resulted in a shape model with a $\chi^{2}=51$. The $\chi^{2}$ corresponds to the square of the difference between the observed and synthetic images, given in units of the instrumental noise without taking into account possible residual noise from the deconvolution process (Capanna et al. 2013). Then, we searched for the best spin solution by testing a grid of values around the initial ADAM solution. We found spin-vector coordinates $\left([\lambda ; \beta]=\left[35^{\circ} ;-10^{\circ}\right]\right.$ in ECJ2000) close to the values used initially $\left(\left[34^{\circ} ;-9^{\circ}\right]\right.$, Viikinkoski et al. 2018). A second MPCD run was performed using this new spin but resulted in similar $\chi^{2}$ and volume. The final step was to recompute the best-fitting PSF, this time with the MPCD model. As a significantly different FWHM was retrieved (1.5 pixels), we performed a last MPCD run that gave a final shape model with a $\chi^{2}=43$.

\section{Results}

\subsection{Comparison of the ADAM and MPCD models}

In order to compare the two methods, the OASIS synthetic images were generated using the same PSF and pole direction for each model. A comparison between the observed images and the corresponding synthetic images of both MPCD and ADAM models is given in Fig. 1, along with the residual images. The two models are remarkably similar. They have a volume difference of $2.6 \%$ and the distribution of their radial differences measured along the normal direction has a mean of $-0.7 \mathrm{~km}$ with a standard deviation of $\sim 2 \mathrm{~km}$ (Fig. A.5). Their $\chi^{2}$ are compared in Table 1 with those of the radar model of Shepard et al. (2017) and the previous ADAM model (without the images of the 2019 apparition), which shows a significant increase in the quality of the fit to the images by the new models.

\subsection{Bulk density}

Both of our shape models feature a slightly smaller volumeequivalent diameter with respect to the model presented in Viikinkoski et al. (2018). Combining our new volume estimates with the average of available mass estimates determined in Viikinkoski et al. (2018) yields a density of $4.20 \pm 0.60 \mathrm{~g} \mathrm{~cm}^{-3}$ for MPCD and $4.10 \pm 0.61 \mathrm{~g} \mathrm{~cm}^{-3}$ for ADAM. We did not include the lower mass $\left(1.5 \times 10^{19} \mathrm{~kg}\right)$ derived by Siltala \& Granvik (2020) in the mass estimation as we consider this value to be an outlier. The physical characteristics of our new models compared to those of previous works are reported in Table 1.

\subsection{Shape analysis}

The analysis of Psyche's shape was first performed by computing the radial differences between Psyche's shape model and its best-fitting ellipsoid, leading to a (model - ellipsoid) average residuals of $3.6 \%$ relative to the mean radius (computed as the square root of the reduced $\chi^{2}$ ). We then calculated the sphericity index of Psyche following the same approach as in Vernazza et al. (2019), and obtained a value of $\psi=0.9548$. For 
Table 1. Comparison of the physical parameters of Psyche of previous works from Viikinkoski et al. (2018) and augmented with the values derived in this study with the ADAM and MPCD methods.

\begin{tabular}{lccccc}
\hline \hline Parameter & S17 & D18 & V18 & This work, ADAM & This work, MPCD \\
\hline$D(\mathrm{~km})$ & $226 \pm 23$ & $223 \pm 2$ & $226 \pm 5$ & $224 \pm 5$ & $222 \pm 4$ \\
$\lambda\left(\left(^{\circ}\right)\right.$ & $34 \pm 34$ & $32 \pm 3$ & $34 \pm 3$ & $35 \pm 2$ & $35 \pm 2$ \\
$\beta\left({ }^{\circ}\right)$ & $-7 \pm 5$ & $-8 \pm 3$ & $-9 \pm 3$ & $-9 \pm 2$ & $-10 \pm 2$ \\
$P(\mathrm{~h})$ & $4.195948(1)$ & $4.195951(2)$ & $4.195948(1)$ & $4.195948(1)$ & $4.195948(1)$ \\
$a(\mathrm{~km})$ & $279 \pm 27$ & $274 \pm 2$ & $290 \pm 5$ & $286 \pm 5$ & $278 \pm 5(277)$ \\
$b(\mathrm{~km})$ & $232 \pm 23$ & $231 \pm 4$ & $245 \pm 5$ & $240 \pm 5$ & $232 \pm 6(238)$ \\
$c(\mathrm{~km})$ & $189 \pm 19$ & $176 \pm 3$ & $170 \pm 8$ & $172 \pm 4$ & $164 \pm 4(168)$ \\
$a / b$ & $1.17 \pm 0.17$ & $1.18 \pm 0.02$ & $1.18 \pm 0.03$ & $1.19 \pm 0.03$ & $1.20 \pm 0.04(1.16)$ \\
$b / c$ & $1.21 \pm 0.17$ & $1.31 \pm 0.03$ & $1.44 \pm 0.07$ & $1.40 \pm 0.04$ & $1.41 \pm 0.05(1.42)$ \\
$a / c$ & $1.50 \pm 0.17$ & $1.56 \pm 0.02$ & $1.71 \pm 0.07$ & $1.66 \pm 0.04$ & $1.70 \pm 0.06(1.69)$ \\
$m\left(10^{18} \mathrm{~kg}\right)$ & $27.2 \pm 7.5$ & $24.3 \pm 3.5$ & $24.1 \pm 3.2$ & $24.1 \pm 3.2$ & $24.1 \pm 3.2$ \\
$\left.\rho(\mathrm{g} \mathrm{cm})^{-3}\right)$ & $4.5 \pm 1.4$ & $4.16 \pm 0.64$ & $3.99 \pm 0.61$ & $4.10 \pm 0.61$ & $4.20 \pm 0.60$ \\
\hline$\chi^{2}$ & 167 & & 94 & 54 & 43 \\
\hline
\end{tabular}

Notes. Listed parameters are the volume equivalent-diameter $D$, the dimensions along the major axes $(a, b, c)$, the sidereal period $P$, the pole ecliptic longitude $\lambda$ and latitude $\beta$, the mass $m$, and the bulk density $\rho$ with uncertainties reported at $1 \sigma$. The dimensions of the best-fit ellipsoid (see Sect. 4.3) are indicated between parentheses in the MPCD column. The $\chi^{2}$ computed with OASIS from our set of SPHERE images is also indicated when possible.

References. S17: Shepard et al. (2017), D18: Drummond et al. (2018), V18: Viikinkoski et al. (2018).

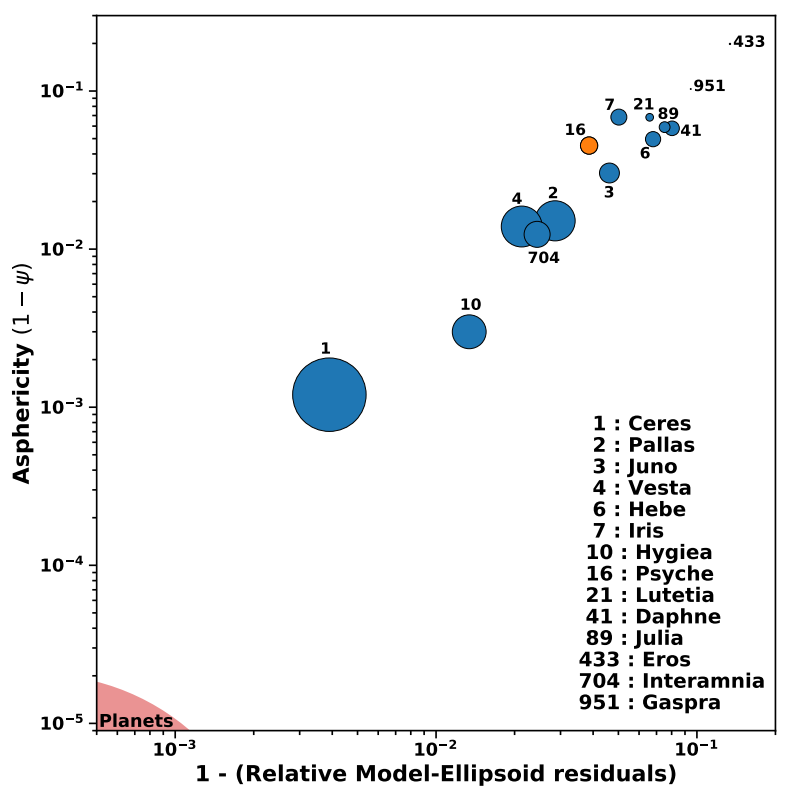

Fig. 2. Asphericity of small solar system objects as a function of their departure from their best-fit ellipsoid. The dot sizes are proportionate to the objects mean radius. The red region on the bottom left of the figure indicates typical values for the terrestrial planets.

comparison, we repeated this process for the previously studied targets of our large program, as well as for the terrestrial planets, dwarf planet Ceres, and a few other asteroids visited in situ by space missions (Fig. 2). Psyche's shape appears to be intermediate between those of larger asteroids such as Interamnia or Vesta and those of smaller or similarly sized bodies such as Iris.

The appearance of Psyche is close to an ellipsoidal shape along its spin axis direction and has flat regions on both poles. On the other hand, its shape deviates from an ellipsoid in the other directions because of depression regions along the equator, possibly caused by impact basins. To obtain the 3D shape of the triaxial ellipsoid that represents the primordial shape of Psyche, we clipped those three depressions in the MPCD model
Table 2. Approximate coordinates of the center and sizes of the three equatorial depressions.

\begin{tabular}{lccc}
\hline \hline & A & B & C \\
\hline Latitude $\left[{ }^{\circ}\right]$ & -7 & -7 & 7 \\
Longitude $\left[{ }^{\circ}\right]$ & -90 & 30 & 130 \\
Length $[\mathrm{km}]$ & 50 & 78 & 90 \\
Width $[\mathrm{km}]$ & 44 & 60 & 80 \\
\hline
\end{tabular}

in order to ignore the corresponding vertex in the computation of the best-fit ellipsoid. The result is a triaxial ellipsoid with dimensions $a=277 \mathrm{~km}, b=238 \mathrm{~km}$, and $c=168 \mathrm{~km}$. The three depressions, denoted A, B, and C, appear in the distance map in Fig. A.6 as large regions of negative values. The approximate body-centered latitude and longitude of their centers are reported in Table 2. The presence of such three large cavities $(D>50 \mathrm{~km})$ seems to be incompatible with a pure metallic composition (Marchi et al. 2020). The difference between the equatorial and the pole-on views is highlighted in Fig. A.7, which shows three profiles of the MPCD model along with the best-fit ellipsoid. The mass-deficit region noted $\mathrm{B}$ was already detected in the radar model of Shepard et al. (2017) and is clearly visible in Viikinkoski et al. (2018). A protrusion between the B and C depressions can also be seen in the pole view, potentially owing to compression caused by impacts on both sides.

\section{Discussion}

Both the flatness of Psyche's shape $(a-c \sim 91 \mathrm{~km})$ and its small departure from an ellipsoid open the possibility that Psyche formed at equilibrium and that its shape was subsequently deformed by collisions. We thus investigated whether the shape of Psyche may be close to the equipotential shape of an hydrostatic body. As in previous studies (Rambaux et al. 2015; Park et al. 2016; Vernazza et al. 2019; Hanuš et al. 2020; Marsset et al. 2020), we compared the equipotential shape with the observed shape. First, we computed axisymetric solutions. The computation is based on the numerical resolution of Clairaut's equations 


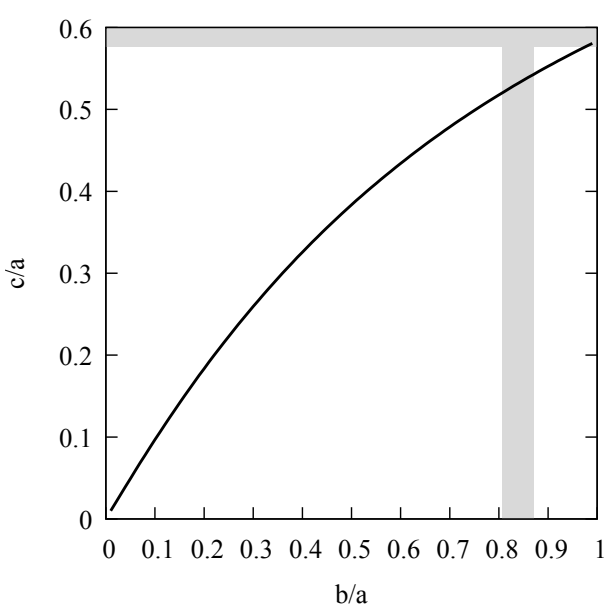

(a)

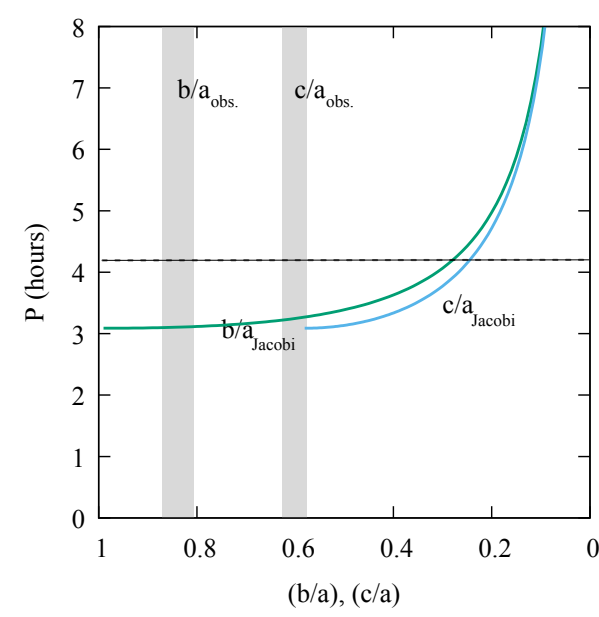

(b)
Fig. 3. (a) Variation of the axial ratios $c / a$ and $b / a$ for the Jacobi ellipsoid. $(b)$ Variations of axial ratios as a function of the rotation period $P$. The gray shaded regions corresponds to the observations of Psyche and uncertainties. The horizontal dashed line in figure $(b)$ indicates Psyche's current rotation period. (see Chambat et al. 2010 and Rambaux et al. 2015). If we assume a homogeneous interior, the density and rotation period of Psyche compatible with a MacLaurin figure at equilibrium leads to an oblate body with $a-c$ of about $50 \mathrm{~km}$, which is significantly less than currently observed. If Psyche was to be differentiated, this value would be even lower. Secondly, we also tested whether Psyche's triaxial shape corresponds to a Jacobi ellipsoid. There is no solution compatible with the present density, rotation, and shape of Psyche. On the other hand, an initial rotation of approximately three hours makes it possible to have a Jacobi figure of equilibrium close to the observed shape as illustrated in Fig. 3. The relative difference is below $10 \%$ for $a / c$ and for $b / a$. This solution could suggest a possible fossil shape acquired when Psyche had a faster rotation.

If Psyche effectively formed at equilibrium, this implies that Psyche's primordial shape was acquired while the body behaved as a fluid. This can be achieved via two different formation scenarios: first, a giant impact while Psyche's interior was already frozen followed by a re-accumulation event as in the case of Hygiea (Vernazza et al. 2019) or, second, a giant impact in the very early solar system while the parent body of Psyche was molten as a consequence of the decay of the short-lived radionuclide ${ }^{26} \mathrm{Al}$. Of great interest, the latter scenario would somewhat correspond to the scenario invoked to explain the formation of the mesosiderite parent body (Scott et al. 2001). In this scenario, the high metal content of Psyche's surface (Shepard et al. 2017) as well as its mesosiderite-like density (Viikinkoski et al. 2018) would be naturally reproduced. We note that in both cases, the impact had to be extremely energetic to lead to a short rotation period of $\sim 3 \mathrm{~h}$, such a rotation period being extremely unusual for a body of that size. While the YORP effect is not significant for objects larger than 30-40 km (Vokrouhlický et al. 2015), later impacts may have affected Psyche's rotation and slowed it down. Future dedicated smoothed-particle hydrodynamics simulations would be useful to test whether the metallic core of a differentiated yet frozen body could be mixed up efficiently with its mantle to explain the high metal content of Psyche's surface.

Acknowledgements. Based on observations collected at the European Organisation for Astronomical Research in the Southern Hemisphere under ESO programme 199.C-0074 (principal investigator: P. Vernazza). P. Vernazza, A. Drouard, M. Ferrais and B. Carry were supported by CNRS/INSU/PNP. J.H and J.D. were supported by grant 18-09470S of the Czech Science Foundation and by the Charles University Research Programme no. UNCE/SCI/023. E.J. is F.R.S.-FNRS Senior Research Associate. The work of TSR was carried out through grant APOSTD/2019/046 by Generalitat Valenciana (Spain). This work was supported by the MINECO (Spanish Ministry of Economy) through grant RTI2018-095076-B-C21 (MINECO/FEDER, UE).

\section{References}

Asphaug, E., \& Reufer, A. 2014, Nat. Geosci., 7, 564

Asphaug, E., Agnor, C. B., \& Williams, Q. 2006, Nature, 439, 155

Beuzit, J. L., Feldt, M., Dohlen, K., et al. 2008, Proc. SPIE, 7014, 701418

Capanna, C., Gesquière, G., Jorda, L., Lamy, P., \& Vibert, D. 2013, Visual Comput., 29, 825

Carry, B. 2012, Planet. Space Sci., 73, 98

Carry, B., Vachier, F., Berthier, J., et al. 2019, A\&A, 623, A132

Chambat, F., Ricard, Y., \& Valette, B. 2010, Geophys. J. Int., 183, 727

Descamps, P., Marchis, F., Berthier, J., et al. 2011, Icarus, 211, 1022

Drummond, J. D., Merline, W. J., Carry, B., et al. 2018, Icarus, 305, 174

Dunham, D., Herald, D., Frappa, E., et al. 2017, Asteroid Occultations, NASA Planetary Data System, Tech. Rep., EAR-A-3-RDR-OCCULTATIONSV15.0

Elkins-Tanton, L.T., Asphaug, E., Bell, J. F., et al. 2017, Lunar Planet. Sci. Conf., 48,1718

Elkins-Tanton, L., Asphaug, E., Bell, J. F., et al. 2020, J. Geophys. Res. Planets, 125, e06296

Fétick, R. J., Jorda, L., Vernazza, P., et al. 2019, A\&A, 623, A6

Fusco, T., Mugnier, L. M., Conan, J.-M., et al. 2003, Proc. SPIE, 4839, 1065

Hanuš, J., Viikinkoski, M., Marchis, F., et al. 2017, A\&A, 601, A114

Hanuš, J., Vernazza, P., Viikinkoski, M., et al. 2020, A\&A, 633, A65

Hapke, B. 1981, J. Geophys. Res., 86, 3039

Johnson, B. C., Sori, M. M., \& Evans, A. J. 2020, Nat. Astron., 4, 4

Jorda, L., Spjuth, S., Keller, H.U., Lamy, P., \& Llebaria, A. 2010, SPIE Conf. Ser., 7533, 753311

Jorda, L., Gaskell, R., Capanna, C., et al. 2016, Icarus, 277, 257

Marchi, S., Durda, D. D., Polanskey, C. A., et al. 2020, J. Geophys. Res. (Planets), 125, e05927

Marsset, M., Brož, M., Vernazza, P., et al. 2020, Nat. Astron., 4, 569

Matter, A., Delbo', M., Carry, B., \& Ligori, S. 2013, Icarus, 226, 419

Mugnier, L. M., Fusco, T., \& Conan, J.-M. 2004, JOSA A, 21, 1841

Park, R., Konopliv, A., Bills, B., et al. 2016, EGU General Assembly Conf. Abstr., 18, 8395

Rambaux, N., Chambat, F., \& Castillo-Rogez, J. C. 2015, A\&A, 584, A127

Scheeres, D. J., Britt, D., Carry, B., \& Holsapple, K. A. 2015, Asteroid Inter. Morphol., 745

Scott, E. R. D., Haack, H., \& Love, S. G. 2001, Meteorit. Planet. Sci., 36, 869

Shepard, M. K., Richardson, J., Taylor, P. A., et al. 2017, Icarus, 281, 388

Siltala, L., \& Granvik, M. 2020, A\&A, 633, A46

Thalmann, C., Schmid, H.M., Boccaletti, A, et al. 2008, Proc. SPIE, 7014, $70143 \mathrm{~F}$

Vernazza, P., Brož, M., Drouard, A., et al. 2018, A\&A, 618, A154

Vernazza, P., Jorda, L., Ševeček, P., et al. 2019, Nat. Astron., 4, 136

Viikinkoski, M., Kaasalainen, M., \& Durech, J. 2015, A\&A, 576, A8

Viikinkoski, M., Vernazza, P., Hanuš, J., et al. 2018, A\&A, 619, L3

Vokrouhlický, D., Bottke, W. F., Chesley, S. R., Scheeres, D. J., \& Statler, T. S 2015 , in The Yarkovsky and YORP Eects, eds. P. Michel, F. E. DeMeo, \& W. F. Bottke, 509 


\section{Appendix A: Additional figures and tables}
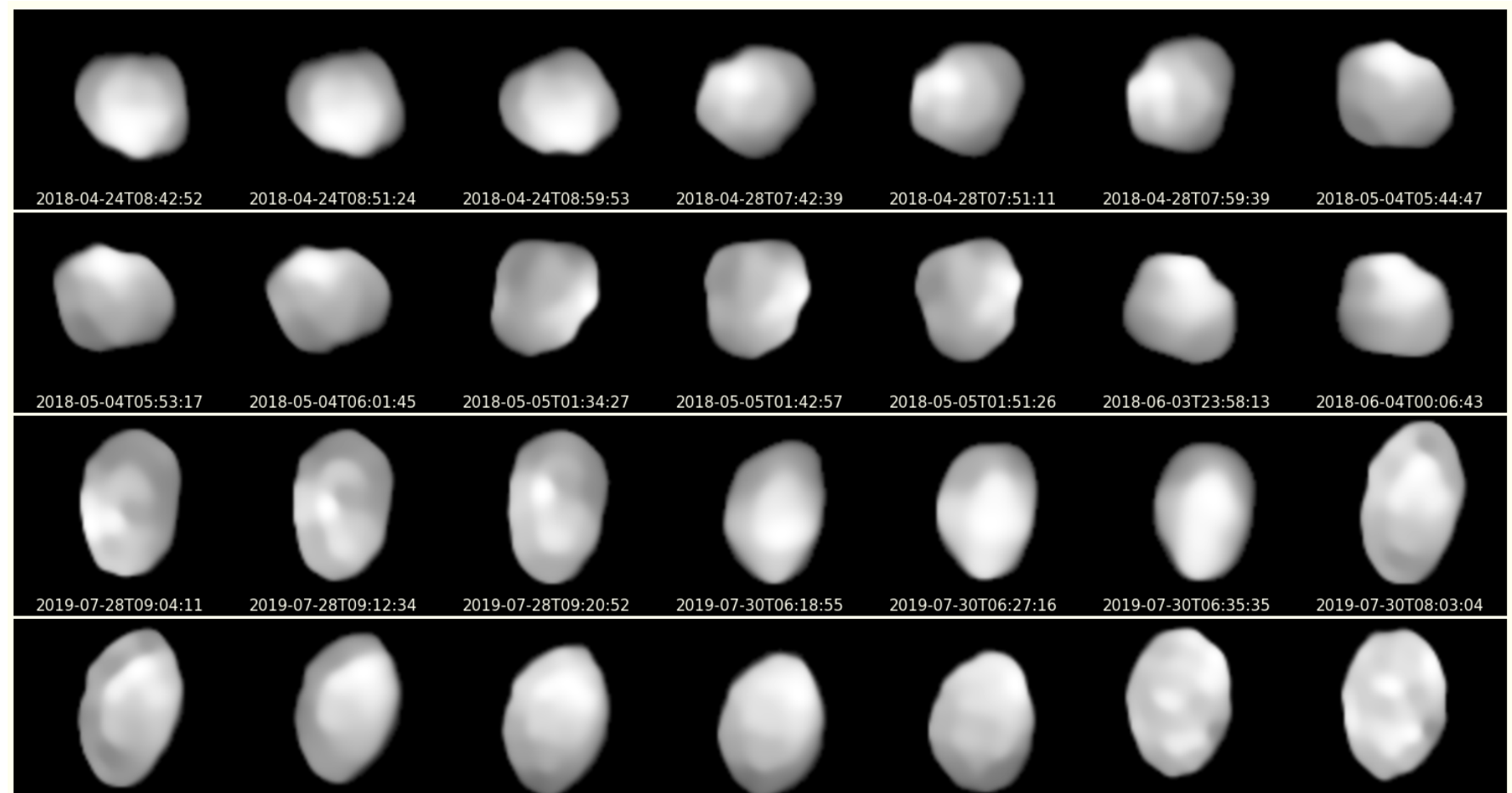

-07-28T09:20:52

9-07-30T06:18:55
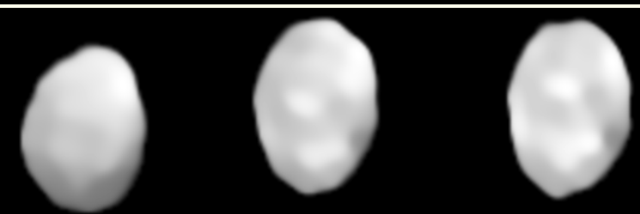

2019-07-30T08:11:26

2019-07-30T08:19:45

2019-08-03T04:39:39

2019-08-03T04:47:59

2019-08-03T04:56:19

2019-08-05T07:43:16

2019-08-05T07:51:38
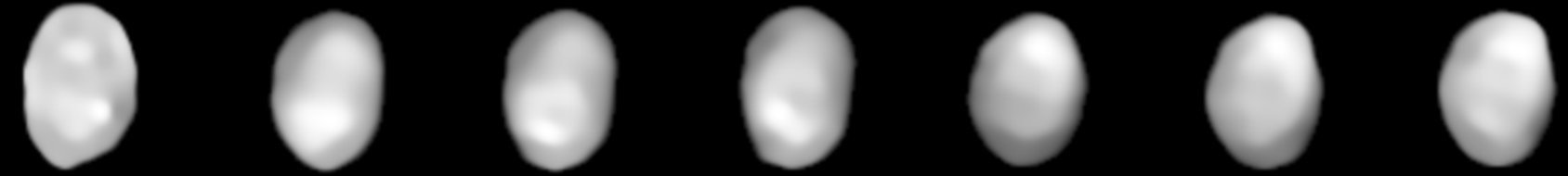

2019-08-05T07:59:58

2019-08-06T02:34:21

2019-08-06T02:42:42

2019-08-06T02:51:01

2019-08-06T04:10:56

2019-08-06T04:19:18

2019-08-06T04:27:36

Fig. A.1. Thirty-five VLT/SPHERE/ZIMPOL images of Psyche selected for our shape reconstruction. The images were obtained at 12 different epochs between April 2018 and August 2019 and deconvolved with the Mistral algorithm.

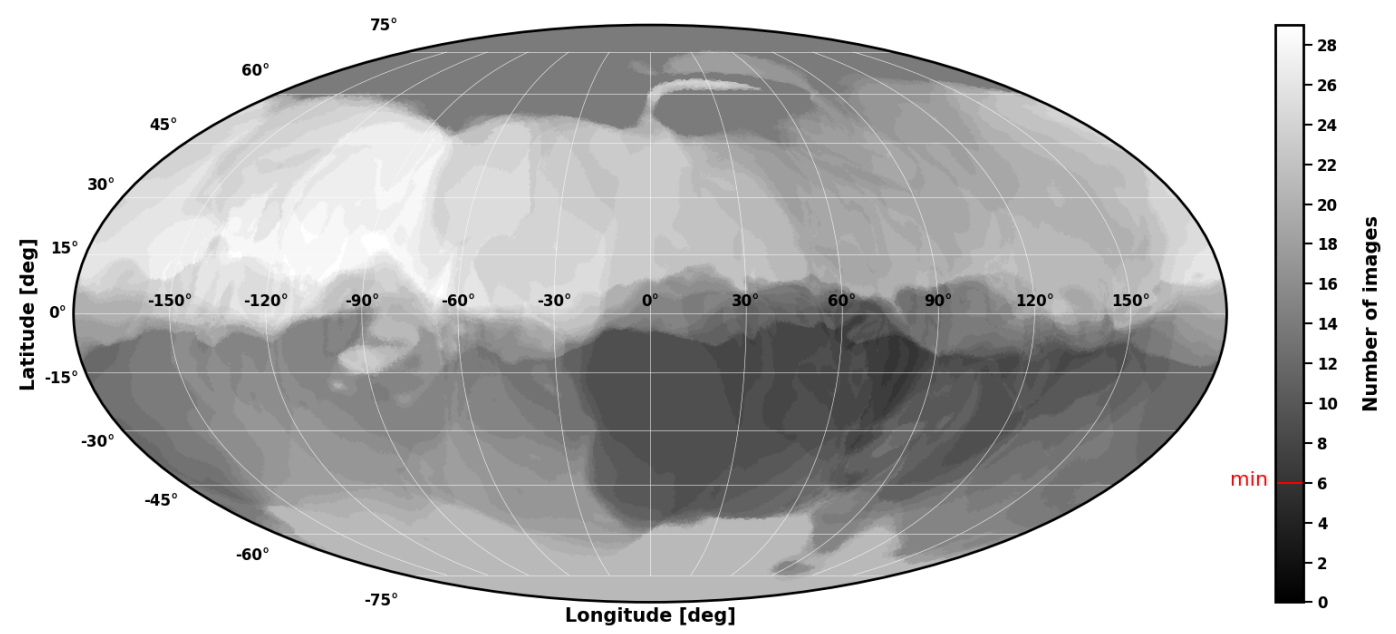

Fig. A.2. Coverage map of the surface of Psyche. The number of times each facet of our shape model has been imaged is represented by different gray levels (zero would be black). Of our total number of 35, the maximum number of images covering a given facet is 29 and the minimum is 6 , meaning a $100 \%$ surface coverage. 
M. Ferrais et al.: Asteroid (16) Psyche's primordial shape: A possible Jacobi ellipsoid

Table A.1. Observational circumstances for each selected observation.

\begin{tabular}{lccccc}
\hline \hline Date & UT & $\begin{array}{c}\text { Exp } \\
\text { (s) }\end{array}$ & Airmass & $\begin{array}{c}\alpha \\
\left({ }^{\circ}\right)\end{array}$ & $\begin{array}{c}D_{\mathrm{a}} \\
\left({ }^{\prime \prime}\right)\end{array}$ \\
\hline $2018-04-24$ & $08: 42: 52$ & 245 & 1.30 & 5.8 & 0.136 \\
$2018-04-24$ & $08: 51: 24$ & 245 & 1.34 & 5.8 & 0.136 \\
$2018-04-24$ & $08: 59: 53$ & 245 & 1.39 & 5.8 & 0.136 \\
$2018-04-28$ & $07: 42: 39$ & 245 & 1.16 & 4.5 & 0.137 \\
$2018-04-28$ & $07: 51: 11$ & 245 & 1.19 & 4.5 & 0.137 \\
$2018-04-28$ & $07: 59: 39$ & 245 & 1.21 & 4.5 & 0.137 \\
$2018-05-04$ & $05: 44: 47$ & 245 & 1.03 & 2.6 & 0.138 \\
$2018-05-04$ & $05: 53: 17$ & 245 & 1.03 & 2.6 & 0.138 \\
$2018-05-04$ & $06: 01: 45$ & 245 & 1.04 & 2.6 & 0.138 \\
$2018-05-05$ & $01: 34: 27$ & 245 & 1.59 & 2.3 & 0.139 \\
$2018-05-05$ & $01: 42: 57$ & 245 & 1.53 & 2.3 & 0.139 \\
$2018-05-05$ & $01: 51: 26$ & 245 & 1.48 & 2.3 & 0.139 \\
$2018-06-03$ & $23: 58: 13$ & 245 & 1.34 & 8.7 & 0.135 \\
$2018-06-04$ & $00: 06: 43$ & 245 & 1.30 & 8.7 & 0.135 \\
$2019-07-28$ & $09: 04: 11$ & 240 & 1.57 & 4.2 & 0.181 \\
$2019-07-28$ & $09: 12: 34$ & 240 & 1.64 & 4.2 & 0.181 \\
$2019-07-28$ & $09: 20: 52$ & 240 & 1.72 & 4.2 & 0.181 \\
$2019-07-30$ & $06: 18: 55$ & 240 & 1.04 & 3.5 & 0.181 \\
$2019-07-30$ & $06: 27: 16$ & 240 & 1.05 & 3.5 & 0.181 \\
$2019-07-30$ & $06: 35: 35$ & 240 & 1.06 & 3.5 & 0.181 \\
$2019-07-30$ & $08: 03: 04$ & 240 & 1.28 & 3.4 & 0.181 \\
$2019-07-30$ & $08: 11: 26$ & 240 & 1.32 & 3.4 & 0.181 \\
$2019-07-30$ & $08: 19: 45$ & 240 & 1.36 & 3.4 & 0.181 \\
$2019-08-03$ & $04: 39: 39$ & 240 & 1.02 & 1.8 & 0.182 \\
$2019-08-03$ & $04: 47: 59$ & 240 & 1.02 & 1.8 & 0.182 \\
$2019-08-03$ & $04: 56: 19$ & 240 & 1.01 & 1.8 & 0.182 \\
$2019-08-05$ & $07: 43: 16$ & 240 & 1.32 & 1.0 & 0.183 \\
$2019-08-05$ & $07: 51: 38$ & 240 & 1.35 & 1.0 & 0.183 \\
$2019-08-05$ & $07: 59: 58$ & 240 & 1.40 & 1.0 & 0.183 \\
$2019-08-06$ & $02: 34: 21$ & 240 & 1.20 & 0.7 & 0.183 \\
$2019-08-06$ & $02: 42: 42$ & 240 & 1.17 & 0.7 & 0.183 \\
$2019-08-06$ & $02: 51: 01$ & 240 & 1.15 & 0.7 & 0.183 \\
$2019-08-06$ & $04: 10: 56$ & 240 & 1.03 & 0.7 & 0.183 \\
$2019-08-06$ & $04: 19: 18$ & 240 & 1.02 & 0.7 & 0.183 \\
$2019-08-06$ & $04: 27: 36$ & 240 & 1.02 & 0.7 & 0.183 \\
\hline & & & & & \\
\hline
\end{tabular}

Notes. For each of the 35 images selected out of the 60 available, the table gives the epoch, exposure time, airmass, phase angle $\alpha$, and angular diameter $D_{\mathrm{a}}$. All images were acquired with VLT/SPHERE using the N_R photometric filter.

Table A.2. Observers of the stellar occultation by Psyche on 24 October 2019.

\begin{tabular}{l}
\hline \hline Observer \\
\hline D. Palmer, La Cueva, NM \\
P. Maley, Camp Verde, AZ \\
D. Dunham, J. Dunham, Nothing, AZ \\
D. Dunham, J. Dunham, Wikieup, AZ \\
D. Dunham, J. Dunham, Kingman, AZ \\
T. George, Scottsdale, AZ \\
S. Herchak, Mesa, AZ \\
R. Jones, Twentynine Palms, CA \\
R. Reaves, Parker, AZ \\
D. Stanbridge, D. Dunham, Queen Valley, AZ \\
D. Stanbridge, D. Dunham, Gold Canyon, AZ \\
W. Thomas, Tempe, AZ \\
T. Blank, P. Yeargain, Wickenburg, AZ \\
R. Wasson, Anza, CA \\
\hline
\end{tabular}
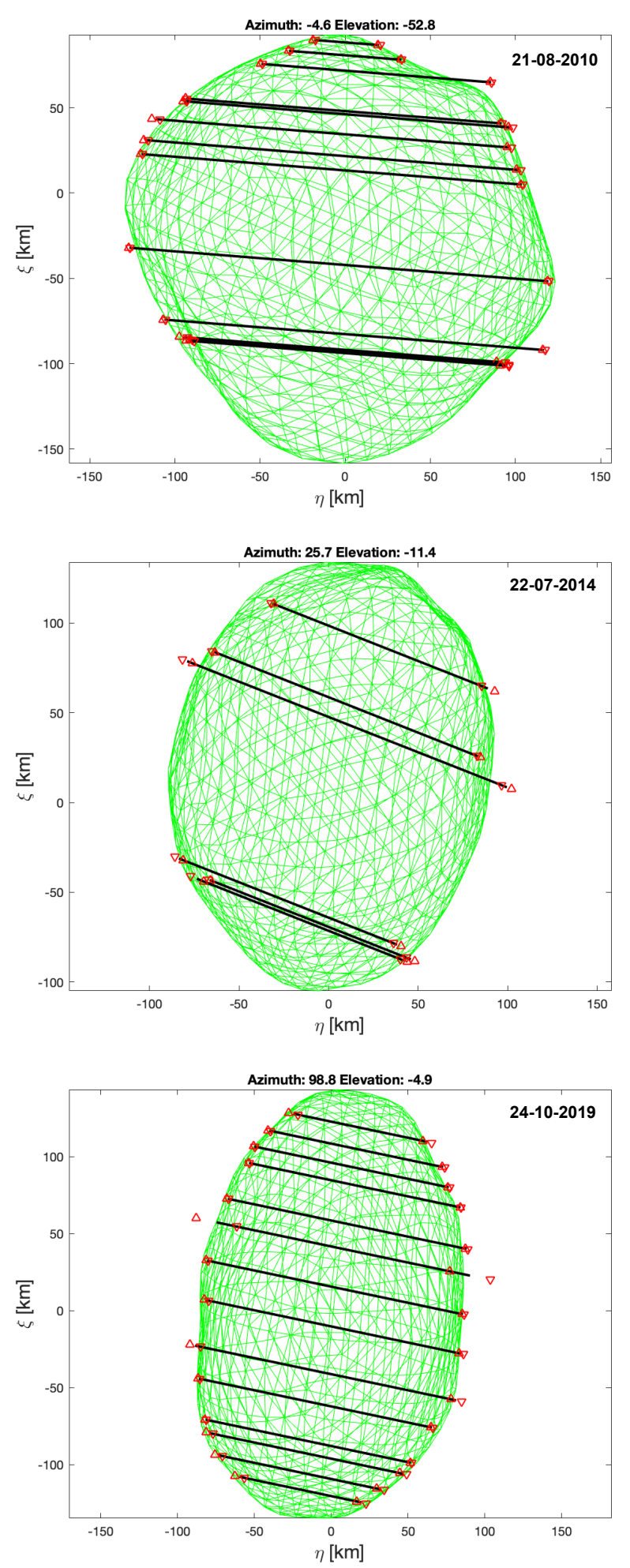

Fig. A.3. Three stellar occultations by Psyche compared to the ADAM model. Disappearance and reappearance timings, as well as their uncertainties, are indicated by red triangles. The disagreement between the model and a few chords of the 2010 and 2014 occultations is probably because those chords are wrong or have underestimated uncertainties. Only the 2019 occultation was used in the ADAM shape modeling. 


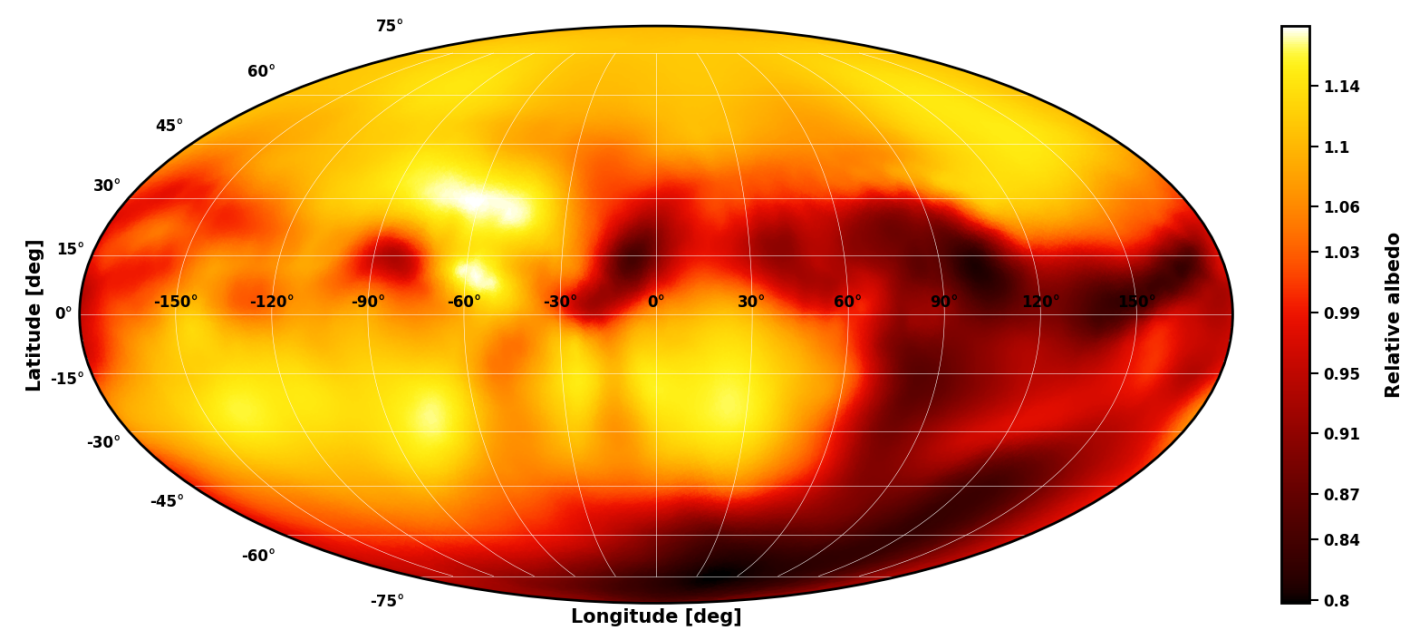

Fig. A.4. Relative albedo map distribution derived with ADAM. The bright region visible in the images of 2018 (Fig. A.1), called Panthia in Viikinkoski et al. (2018), is visible around longitude $\sim-60^{\circ}$ in the north hemisphere. Other albedo features visible in multiple images of the 2019 apparition include one bright spot on 28 July 2019 and 6 August 2019 at (longitude, latitude) $=\sim\left(-60^{\circ},-10^{\circ}\right)$ and two bright spots on 5 August 2019 at $\sim\left(120^{\circ},-15^{\circ}\right)$ and $\sim\left(160^{\circ},-10^{\circ}\right)$.

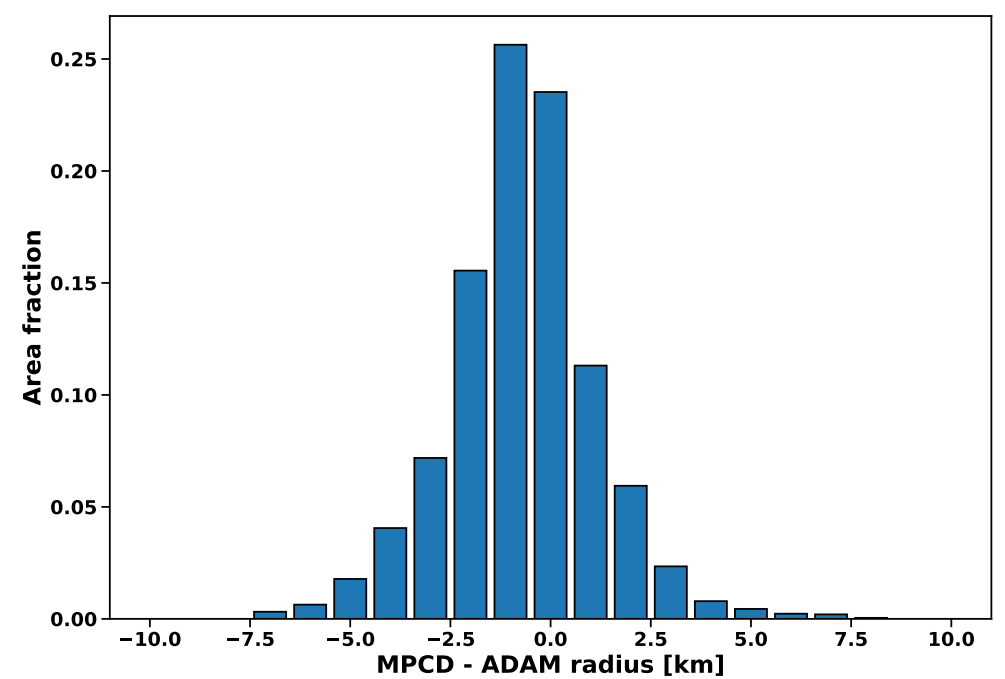

Fig. A.5. Distribution of the distances measured along the normal direction between the MPCD and ADAM models.

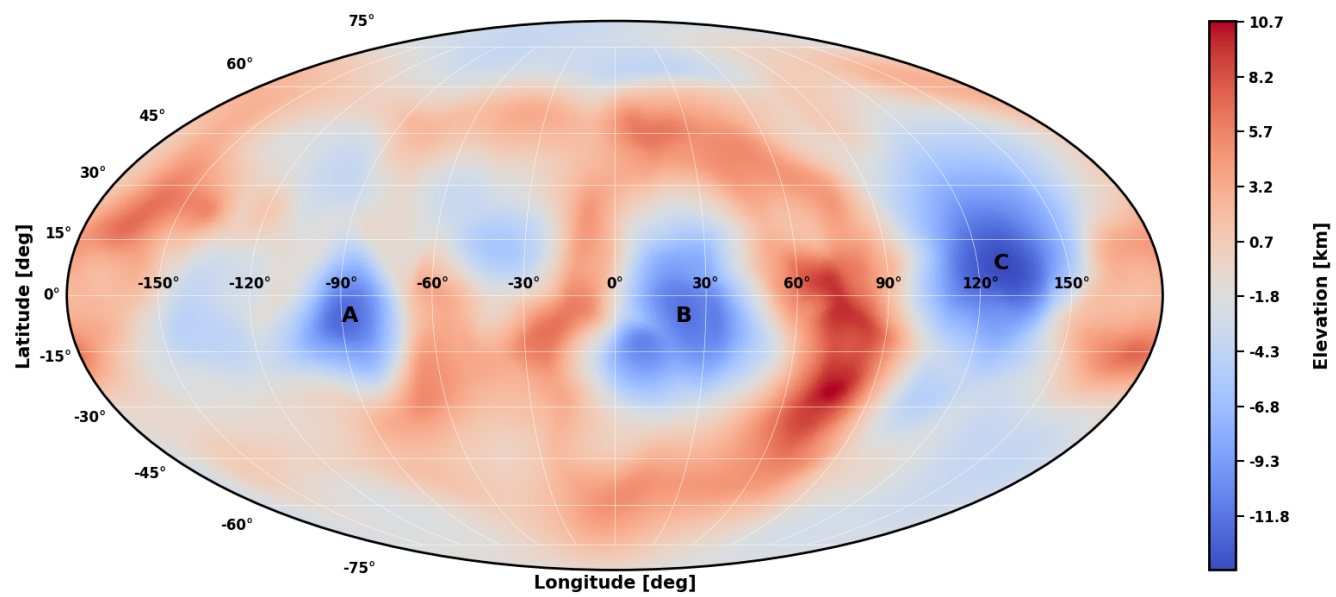

Fig. A.6. Topography of Psyche in kilometers with respect to its best-fit ellipsoid ( $a=277 \mathrm{~km}, b=238 \mathrm{~km}, c=168 \mathrm{~km})$ after clipping the 3 depressions $\mathrm{A}, \mathrm{B}$, and $\mathrm{C}$. 
M. Ferrais et al.: Asteroid (16) Psyche's primordial shape: A possible Jacobi ellipsoid
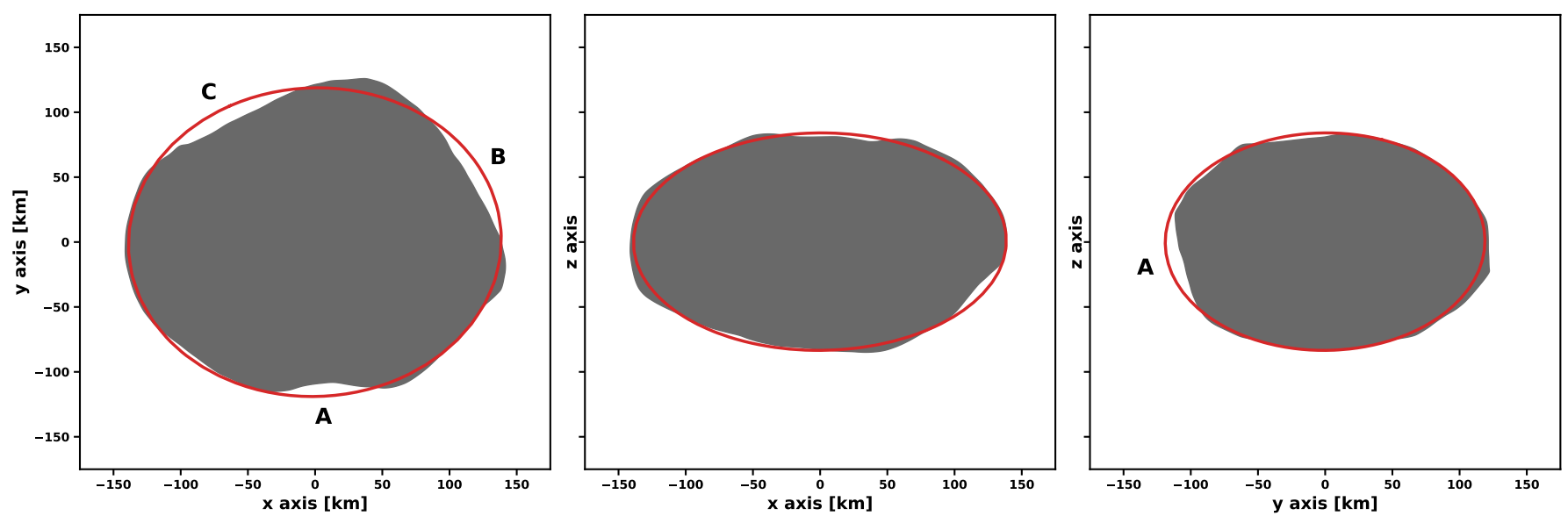

Fig. A.7. Profiles of the MPCD model and of its triaxial best-fit ellipsoid with a pole-on view (left) and two equatorial views (center and right). The $x, y, z$ axes are aligned along the principal axes of inertia (assuming an homogeneous interior). 